QCD Evolution Workshop 2013

International Journal of Modern Physics: Conference Series

Vol. 25 (2014) 1460040 (8 pages)

(C) The Author

DOI: $10.1142 / \mathrm{S} 2010194514600404$

\title{
DOUBLE PARTON FRAGMENTATION FUNCTION AND ITS EVOLUTION IN QUARKONIUM PRODUCTION*
}

\author{
ZHONG-BO KANG \\ Theoretical Division, Los Alamos National Laboratory \\ Los Alamos, New Mexico 87545, USA \\ zkang@lanl.gov
}

Published 2 January 2014

\begin{abstract}
We summarize the results of a recent study on a new perturbative QCD factorization formalism for the production of heavy quarkonia of large transverse momentum $p_{T}$ at collider energies. Such a new factorization formalism includes both the leading power (LP) and next-to-leading power (NLP) contributions to the cross section in the $m_{Q}^{2} / p_{T}^{2}$ expansion for heavy quark mass $m_{Q}$. For the NLP contribution, the so-called double parton fragmentation functions are involved, whose evolution equations have been derived. We estimate fragmentation functions in the non-relativistic QCD formalism, and found that their contribution reproduce the bulk of the large enhancement found in explicit NLO calculations in the color singlet model. Heavy quarkonia produced from NLP channels prefer longitudinal polarization, in contrast to the single parton fragmentation function. This might shed some light on the heavy quarkonium polarization puzzle.
\end{abstract}

Keywords: Double parton fragmentation function; heavy quarkonium production; QCD evolution

PACS numbers: 12.38.Bx, 12.39.St, 13.87.Fh, 14.40.Pq

\section{Introduction}

In these proceedings we present the results of Refs. [1, 2], where a perturbative QCD factorization formalism for the production of heavy quarkonium of large transverse momentum $p_{T}$ at collider energies is proposed and it includes both leading power (LP) and next-to-leading power (NLP) contributions to the cross section in the $m_{Q}^{2} / p_{T}^{2}$ expansion for heavy quark mass $m_{Q}$. The NLP contributions involve the so-called double parton or heavy quark pair fragmentation function. We provide the corresponding operator definitions for these fragmentation functions and derive their QCD evolution equations. We discuss the relative role of LP and NLP contributions to the production rate and polarization of heavy quarkonia.

*This is an Open Access article published by World Scientific Publishing Company. It is distributed under the terms of the Creative Commons Attribution 3.0 (CC-BY) License. Further distribution of this work is permitted, provided the original work is properly cited. 
Almost forty years after the discovery of the $J / \psi[3]$, the production of heavy quarkonia remains a key subject in strong interaction physics. ${ }^{4}$ There are various formalisms on the market, which deal with the heavy quarkonium production in hadronic collisions, such as the color singlet model (CSM), the color evaporation model (CEM), and non-relativistic QCD (NRQCD). The current status of theory and experiment has been summarized very recently by the quarkonium working group. ${ }^{4}$ So far the most successful formalism in terms of phenomenology is the NRQCD formalism. ${ }^{5,6}$ In particular, the ability of small color-octet production matrix elements to provide good fits to the high $p_{T}$-dependence of collider cross section data for both $J / \psi$ and $\Upsilon$ is an impressive success. On the other hand, polarization remains a challenge, along with the surprisingly high level of associate production in electron-positron annihilation. ${ }^{4}$

At the same time, large next-to-leading order (NLO) and potentially large nextto-next-to-leading order (NNLO) corrections to high- $p_{T} \operatorname{cross}_{\text {sections }}{ }^{7-9}$ in the color-singlet channel have attracted attention: the NLO contribution is more than a factor of 10 larger than the LO result, and the estimated NNLO contribution suggests that it may be another factor of 10 larger than the NLO result. Such huge enhancement from high order corrections naturally raises the question on whether the perturbative expansion in powers of $\alpha_{s}$ in color-singlet channel is reliable. We find that such unstable perturbative series is due to a mixture of $p_{T}$ power expansion and the $\alpha_{s}$ expansion. In other words, the higher power in terms of $\alpha_{s}$ is not necessarily the higher power in terms of $p_{T}$ expansion.

We thus propose a two step expansion: perform the expansion of the production rate of the heavy quark pairs first in the large scale $p_{T}$ and then in the coupling constant $\alpha_{s}$. We present a perturbative QCD (pQCD) factorization formalism, accurate to the first nonleading power in $m_{Q}^{2} / p_{T}^{2}$ that incorporates both leading power gluon fragmentation and direct production of heavy quarks at short distances with subsequent fragmentation, as illustrated in Fig. $1[1,2]$. When $p_{T} \gg m_{Q}$, the rate to produce a gluon (or in general a single parton) at distance scale $1 / p_{T}$, which
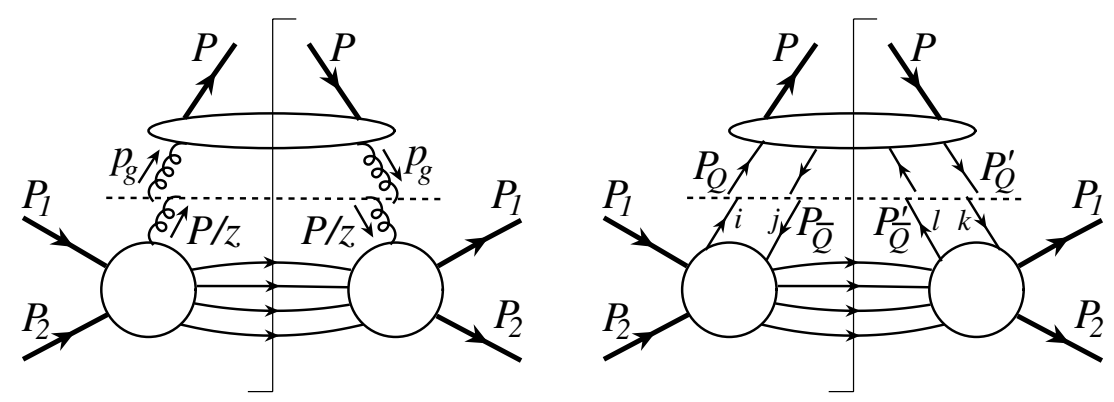

Fig. 1. $J / \psi$ production through a single parton (a gluon) fragmentation (left) and fragmentation of a heavy quark pair (right). 
fragments into a heavy quark pair and thence into a physical quarkonium, is characterized by a $p_{T}^{-4}$ behavior and usually referred to as the LP contribution. The perturbative production of a collinear heavy quark pair directly at the short distance scale $1 / p_{T}$ is suppressed by $1 / p_{T}^{2}$ relative to the production rate of a single parton at the same $p_{T}$, which is referred to as the NLP contribution. We find that the probability for such a heavy quark pair to evolve into a heavy quarkonium is naturally enhanced compared to that of a single parton, and thus can promote the NLP channel to phenomenological interest, despite its suppression by two powers of $p_{T}$.

\section{Factorization Formalism and QCD Evolution of the Fragmentation Functions}

For the heavy quarkonium production in hadronic collisions at the collider energies,

$$
A\left(P_{1}\right)+B\left(P_{2}\right) \rightarrow H(p)+X
$$

where $p$ is the momentum of the produced quarkonium, our proposed factorization formalism is given by

$$
\begin{aligned}
d \sigma_{A+B \rightarrow H+X}(p) \approx & \sum_{f} d \hat{\sigma}_{A+B \rightarrow f+X}\left(p_{f}=p / z, \mu\right) \otimes D_{H / f}\left(z, m_{Q}, \mu\right) \\
& +\sum_{[Q \bar{Q}(\kappa)]} d \hat{\sigma}_{A+B \rightarrow[Q \bar{Q}(\kappa)]+X}\left(p(1 \pm \zeta) / 2 z, p\left(1 \pm \zeta^{\prime}\right) / 2 z, \mu\right) \\
& \otimes \mathcal{D}_{H /[Q \bar{Q}(\kappa)]}\left(z, \zeta, \zeta^{\prime}, m_{Q}, \mu\right),
\end{aligned}
$$

The first (second) term on the right-hand side gives the contribution of LP (NLP) in $m_{Q}^{2} / p_{T}^{2}$. In the first term, $d \hat{\sigma}_{A+B \rightarrow f+X}\left(p_{f}=p / z, \mu\right)$ is the cross section to produce an on-shell parton of flavor $f$ at short distances, which contains all of the information about the incoming state and includes convolutions with parton distributions when $A$ or $B$ is a hadron. $D_{H / f}\left(z, m_{Q}, \mu\right)$ is the usual single parton fragmentation function, representing the transition from a single parton $f$ to a heavy quarkonium state $H$ with momentum fraction $z$ [10]. On the other hand, $d \hat{\sigma}_{A+B \rightarrow[Q \bar{Q}(\kappa)]+X}\left(p(1 \pm \zeta) / 2 z, p\left(1 \pm \zeta^{\prime}\right) / 2 z, \mu\right)$ describes production of an on-shell collinear heavy quark pair, with $\mathcal{D}_{H /[Q \bar{Q}(\kappa)]}\left(z, \zeta, \zeta^{\prime}, m_{Q}, \mu\right)$ the double parton (heavy quark pair) fragmentation function, representing the transition from a heavy quark pair to the heavy quarkonium state $H$. The momentum fractions $z, \zeta$ and $\zeta^{\prime}$ are defined as

$$
\begin{array}{ll}
p_{Q}^{+}=p^{+} \frac{1+\zeta}{2 z}, & p_{\bar{Q}}^{+}=p^{+} \frac{1-\zeta}{2 z} \\
p_{Q}^{+}=p^{+} \frac{1+\zeta^{\prime}}{2 z}, & p_{\bar{Q}}^{\prime+}=p^{+} \frac{1-\zeta^{\prime}}{2 z} .
\end{array}
$$


In other words, $z$ measures the fractional momentum of the pair carried by the observed quarkonium, while $\zeta$ and $\zeta^{\prime}$ characterize the sharing of the pair's momentum between the heavy quark and antiquark on either side of the cut in the figure.

The single parton fragmentation functions $D_{H / f}\left(z, m_{Q}, \mu\right)$ has the usual definition, and it is given in Ref.[10]. On the other hand, the operator definition for $\mathcal{D}_{H /[Q \bar{Q}(\kappa)]}\left(z, \zeta, \zeta^{\prime}, m_{Q}, \mu\right)$ depends on $\kappa$, which represents the pair's color and spin. For the heavy quark pair moving in the " $+z$ " direction with light-cone momentum components, $p^{\mu}=\left(p^{+},\left(2 m_{Q}\right)^{2} / 2 p^{+}, 0_{\perp}\right)$, there are singlet (1) and octet (8) color states, and four spin states described by relativistic Dirac spin projection operators: $\gamma^{+} \gamma_{5} / 4 p^{+}, \gamma^{+} / 4 p^{+}$, and $\gamma^{+} \gamma^{i} / 4 p^{+}$with $i=1,2$, for effective axial vector $(a)$, vector $(v)$, and tensor $(t)$ "currents", respectively. ${ }^{1,2}$ For example, the operator definition of the axial vector/octet fragmentation function can be written as

$$
\begin{aligned}
\mathcal{D}_{H /[Q \bar{Q}(a 8)]} & \left(z, \zeta, \zeta^{\prime}, m_{Q}, \mu\right)=\sum_{X} \int \frac{p^{+} d y^{-}}{2 \pi} \mathrm{e}^{-i\left(p^{+} / z\right) y^{-}} \int \frac{p^{+} d y_{1}^{-} p^{+} d y_{2}^{-}}{(2 \pi)^{2}} \\
& \times \mathrm{e}^{i\left(p^{+} / 2 z\right)(1-\zeta) y_{1}^{-}} \mathrm{e}^{-i\left(p^{+} / 2 z\right)\left(1-\zeta^{\prime}\right) y_{2}^{-}} \\
& \times \frac{4}{\left(N_{c}^{2}-1\right)}\left\langle 0\left|\psi_{i}(0) \frac{\gamma^{+} \gamma_{5}}{4 p^{+}}\left(t^{a}\right)_{i j} \bar{\psi}_{j}\left(y_{2}^{-}\right)\right| H\left(p^{+}\right) X\right\rangle \\
& \times\left\langle H\left(P^{+}\right) X\left|\psi_{l}\left(y^{-}+y_{1}^{-}\right) \frac{\gamma^{+} \gamma_{5}}{4 p^{+}}\left(t^{a}\right)_{l k} \bar{\psi}_{k}\left(y^{-}\right)\right| 0\right\rangle,
\end{aligned}
$$

where we have suppressed gauge links along the minus light cone, inserted between repeated color indices, which provide a gauge invariant definition of the operator. ${ }^{1,2,11}$ The links are in adjoint representation for index $a$, and fundamental representation for $i, j, l, k$. They are reminiscent of hadronic wave functions that connect multiple partons to the hard scattering in the factorized expressions for elastic amplitudes. $^{12}$

Similar to the single parton fragmentation functions, heavy quark pair fragmentation functions like the one defined in Eq. (4) are nonperturbative, but universal. They are boost invariant, and require renormalization. They thus evolve in the usual sense, and depend upon a factorization scale. Such a usual DGLAP-type evolution equations can be written conveniently in a matrix form as follows:

$$
\frac{\partial}{\partial \ln \mu^{2}}\left(\begin{array}{c}
\mathcal{D}_{Q \bar{Q}[v 8]} \\
\mathcal{D}_{Q \bar{Q}[v 1]} \\
\mathcal{D}_{Q \bar{Q}[a 8]} \\
\mathcal{D}_{Q \bar{Q}[a 1]} \\
\mathcal{D}_{Q \bar{Q}[t 8]} \\
\mathcal{D}_{Q \bar{Q}[t 1]}
\end{array}\right)=\frac{\alpha_{s}}{2 \pi}\left(\begin{array}{cccccc}
\mathcal{K} & \mathcal{R} & \mathcal{T}_{1} & \mathcal{T}_{2} & 0 & 0 \\
\widetilde{\mathcal{R}} & \mathcal{S} & \widetilde{\mathcal{T}}_{2} & 0 & 0 & 0 \\
\mathcal{T}_{1} & \mathcal{T}_{2} & \mathcal{K} & \mathcal{R} & 0 & 0 \\
\widetilde{\mathcal{T}}_{2} & 0 & \widetilde{\mathcal{R}} & \mathcal{S} & 0 & 0 \\
0 & 0 & 0 & 0 & \mathcal{K}^{\prime} & \mathcal{R}^{\prime} \\
0 & 0 & 0 & 0 & \widetilde{\mathcal{R}}^{\prime} & \mathcal{S}^{\prime}
\end{array}\right) \otimes\left(\begin{array}{c}
\mathcal{D}_{Q \bar{Q}[v 8]} \\
\mathcal{D}_{Q \bar{Q}[v 1]} \\
\mathcal{D}_{Q \bar{Q}[a 8]} \\
\mathcal{D}_{Q \bar{Q}[a 1]} \\
\mathcal{D}_{Q \bar{Q}[t 8]} \\
\mathcal{D}_{Q \bar{Q}[t 1]}
\end{array}\right)
$$

Direct computations, involving the diagrams shown in Fig. 2 give for the evolution kernels of Eq. (5). These calculations are straightforward though quite subtle 


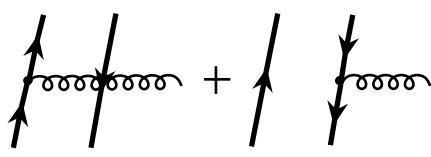

(a)

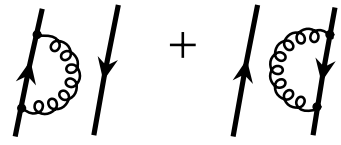

(b)

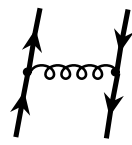

(c)

Fig. 2. Diagrams that evolve quark pair fragmentation in light-cone gauge

at some point and the final results will be presented in a forthcoming paper ${ }^{11}$ with all the details.

\section{Cross Section and Polarization}

It is the fragmentation functions that determine the absolute normalization of perturbative calculations using the factorization formula in Eq. (2), as well as the differences in the production rate between various quarkonium states and their polarizations. Since only pairs with small relative momentum are likely to form bound quarkonia, we may apply the basic NRQCD factorization hypothesis for heavy quarkonium production to these fragmentation functions to reduce the unknown functions to a few universal constants in the form of NRQCD matrix elements,

$$
\begin{gathered}
D_{H / f}\left(z, m_{Q}, \mu\right)=\sum_{c} d_{f \rightarrow[Q \bar{Q}(c)]}\left(z, m_{Q}, \mu\right)\left\langle O_{[Q \bar{Q}(c)]}^{H}\right\rangle \\
\mathcal{D}_{H /[Q \bar{Q}(\kappa)]}\left(z, \zeta, \zeta^{\prime}, m_{Q}, \mu\right)=\sum_{c} d_{[Q \bar{Q}(\kappa)] \rightarrow[Q \bar{Q}(c)]}\left(z, \zeta, \zeta^{\prime}, m_{Q}, \mu\right)\left\langle O_{[Q \bar{Q}(c)]}^{H}\right\rangle
\end{gathered}
$$

where the functions $d$ are calculable, $\mu$ is a factorization scale, and $\left\langle O_{[Q \bar{Q}(c)]}^{H}\right\rangle$ are local NRQCD matrix elements. ${ }^{6}$

We can use this formalism to help understand the source of the surprisingly large corrections to $J / \psi$ production in the color-singlet channel at NLO and NNLO with predominantly longitudinal polarization, in contrast to the small and transversely polarized LO. ${ }^{13,14}$ First, we estimate the $\mathcal{O}\left(\alpha_{s}\right)$ fragmentation functions from the diagrams in Fig. 3, where the upper lines are fixed at $p / 2$ and Dirac indices are contracted with an NRQCD singlet projection with matrix element essentially equivalent to the CSM. For the $[Q \bar{Q}(a 8)]$ state fragmenting into a color singlet ${ }^{3} \mathrm{~S}_{1}$ heavy

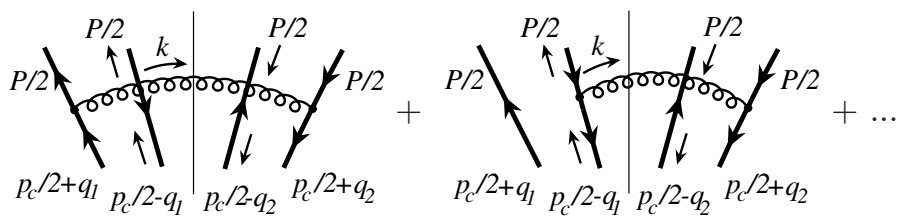

Fig. 3. Leading order Feynman diagrams represent the fragmentation of a heavy quark pair to another heavy quark pair. 


\section{Z.-B. Kang}

quark pair, we have

$$
\begin{aligned}
\mathcal{D}_{[Q \bar{Q}(a 8)] \rightarrow J / \psi}^{L}\left(z, \zeta, \zeta^{\prime}, m_{Q}, \mu\right)= & \frac{1}{2 N_{c}^{2}} \frac{\left\langle O_{1\left({ }^{3} \mathrm{~S}_{1}\right)}^{J / \psi}\right.}{3 m_{c}} \Delta\left(\zeta, \zeta^{\prime}\right) \frac{\alpha_{s}}{2 \pi} z(1-z) \\
& \times\left[\ln (r(z)+1)-\left(1-\frac{1}{1+r(z)}\right)\right], \\
\mathcal{D}_{[Q \bar{Q}(a 8)] \rightarrow J / \psi}^{T}\left(z, \zeta, \zeta^{\prime}, m_{Q}, \mu\right)= & \frac{1}{2 N_{c}^{2}} \frac{\left\langle O_{1\left({ }^{3} \mathrm{~S}_{1}\right)}^{J / \psi}\right.}{3 m_{c}} \Delta\left(\zeta, \zeta^{\prime}\right) \frac{\alpha_{s}}{2 \pi} z(1-z) \\
& \times\left[1-\frac{1}{1+r(z)}\right],
\end{aligned}
$$

where $r(z) \equiv z^{2} \mu^{2} /\left(4 m_{c}^{2}(1-z)^{2}\right)$, and $\mathcal{D}^{L}\left(\mathcal{D}^{T}\right)$ is the pair-fragmentation function with the pair longitudinally (transversely) polarized as in

$$
\mathcal{D}_{[Q \bar{Q}(a 8)] \rightarrow J / \psi}=2 \mathcal{D}_{[Q \bar{Q}(a 8)] \rightarrow J / \psi}^{T}+\mathcal{D}_{[Q \bar{Q}(a 8)] \rightarrow J / \psi}^{L},
$$

with $\mathcal{D}_{[Q \bar{Q}(a 8)] \rightarrow J / \psi}$ the total unpolarized fragmentation function. Function $\Delta\left(\zeta, \zeta^{\prime}\right)$ is given by

$$
\Delta\left(\zeta, \zeta^{\prime}\right)=\frac{1}{4} \sum_{a, b} \delta(\zeta-a(1-z)) \delta\left(\zeta^{\prime}-b(1-z)\right)
$$

with $a, b=-1,1$. In deriving Eqs. (7) and (8), we renormalized the UV divergence by a cutoff $\mu$ on transverse momenta. Quark masses make an IR cutoff on transverse momenta unnecessary. Other renormalization schemes give similar results and will be discussed elsewhere. ${ }^{11}$ It is important to realize that the longitudinal contribution to the heavy quark pair fragmentation function contains the logarithmic piece as in $\ln (r(z)+1)$, while the transverse contribution contains only the power piece. Since the logarithmic piece is dominant at large scale $\mu$ as $r(z) \equiv z^{2} \mu^{2} /\left(4 m_{c}^{2}(1-z)^{2}\right)$, this immediately suggests that heavy quark pair fragmentation function prefers longitudinal polarization.

At the same time, we could compute the short-distance hard parts to produce the relevant heavy quark pair $[Q \bar{Q}(\kappa)]$ state with the corresponding projection operators. ${ }^{11}$ The corresponding LO short-distance hard parts to produce the $[Q \bar{Q}(a 8)]$ state is given in Ref.[1]. For the partonic process $q \bar{q} \rightarrow[Q \bar{Q}(a 8)] g$, the relevant Feynman diagrams are given by Fig. 4. The Feynman diagrams for other processes

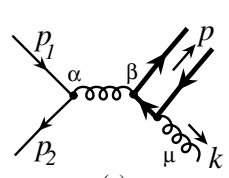

(a)

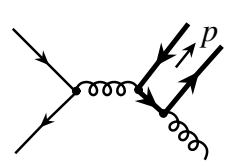

(b)

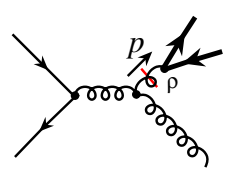

(c)

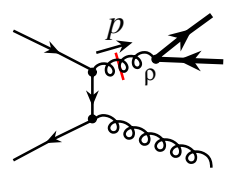

(d)

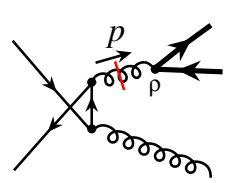

(e)

Fig. 4. Leading order Feynman diagrams for $q \bar{q} \rightarrow Q \bar{Q} g$ subprocess. 
$g q \rightarrow[Q \bar{Q}(a 8)] q$ and $g g \rightarrow[Q \bar{Q}(a 8)] g$ can be easily identified. Finally these short distance hard parts have the following form:

$$
\begin{aligned}
H_{q \bar{q} \rightarrow[Q \bar{Q}(a 8)] g}= & f_{1}\left(\zeta, \zeta^{\prime}\right) \frac{N_{c}^{2}-1}{N_{c}} \frac{2\left(\hat{t}^{2}+\hat{u}^{2}\right)}{\hat{s}^{3}}, \\
H_{g q \rightarrow[Q \bar{Q}(a 8)] q}= & f_{1}\left(\zeta, \zeta^{\prime}\right) \frac{2\left(\hat{s}^{2}+\hat{u}^{2}\right)}{-\hat{t}^{3}}, \\
H_{g g \rightarrow[Q \bar{Q}(a 8)] g}= & \frac{8 N_{c}^{2}}{N_{c}^{2}-1} \frac{\hat{t} \hat{u}}{\hat{s}^{3}}\left(\frac{\hat{t}}{\hat{u}}+1+\frac{\hat{u}}{\hat{t}}\right) \\
& \times\left[f_{1}\left(\zeta, \zeta^{\prime}\right)\left(\frac{\hat{t}}{\hat{u}}+1+\frac{\hat{u}}{\hat{t}}\right)^{3}-f_{2}\left(\zeta, \zeta^{\prime}\right) \frac{5 \hat{s}^{2}}{\hat{t} \hat{u}}\right],
\end{aligned}
$$

where an overall factor of $\left(4 \pi \alpha_{s}^{3} / \hat{s}\right) \delta(\hat{s}+\hat{t}+\hat{u})$ for the invariant cross section $E d \sigma_{A B \rightarrow H} / d^{3} p$ was suppressed, ${ }^{1}$ with $\hat{s}, \hat{t}$, and $\hat{u}$ the standard parton-level Mandelstam variables, and the two special functions $f_{1}\left(\zeta, \zeta^{\prime}\right)$ and $f_{2}\left(\zeta, \zeta^{\prime}\right)$ are given by

$$
\begin{aligned}
& f_{1}\left(\zeta, \zeta^{\prime}\right)=\left[1+\zeta \zeta^{\prime}-\frac{4}{N_{c}^{2}}\right] /\left[\left(1-\zeta^{2}\right)\left(1-\zeta^{\prime 2}\right)\right] \\
& f_{2}\left(\zeta, \zeta^{\prime}\right)=\zeta \zeta^{\prime} /\left[\left(1-\zeta^{2}\right)\left(1-\zeta^{\prime 2}\right)\right] .
\end{aligned}
$$

With both fragmentation functions and LO hard scattering functions, we can compute NLP cross sections that can be compared to NLO CSM cross sections. ${ }^{8,14-16}$ A first result was published in Ref.[1], where we show that indeed the NLP contribution shows the similar enhancement above the LO CSM as the full NLO result in CSM. Similarly, we calculate the $J / \psi$ polarization, as measured by the parameter $\alpha=\left(\sigma^{T}-\sigma^{L}\right) /\left(\sigma^{T}+\sigma^{L}\right)$ in terms of transverse (longitudinal) cross section $\sigma^{T}\left(\sigma^{L}\right)$. As expected, it prefers the longitudinal polarization, which is consistent with that in Refs. $[13,14]$.

\section{Summary}

We have proposed a new perturbative QCD factorization formalism for the heavy quarkonium production at high $p_{T}$ in collider energies, in which one expands the cross sections first in the large scale $p_{T}$, and then in the coupling $\alpha_{s}$. Our formalism includes the first non-leading power contributions in the expansion of $m_{Q}^{2} / p_{T}^{2}$. This next-to-leading power contribution involves the double parton (heavy quark pair) fragmentation functions. We provide their operator definition and study the DGLAP evolution for these new fragmentation functions. We have found that the next-toleading power contributions can be very large and are likely to be longitudinally polarized, in contrast to single parton fragmentation. Our findings might shed light on the long-standing heavy quarkonium polarization puzzle. 


\section{Acknowledgments}

The materials presented here are based on papers with J. -W. Qiu and G. Sterman.

I thank them for collaboration and guidance.

\section{References}

1. Z. -B. Kang, J. -W. Qiu and G. Sterman, Phys. Rev. Lett. 108, 102002 (2012).

2. Z. -B. Kang, J. -W. Qiu and G. Sterman, Nucl. Phys. Proc. Suppl. 214, 39 (2011).

3. J. J. Aubert et al. [E598 Collaboration], Phys. Rev. Lett. 33, 1404 (1974); J. E. Augustin et al. [SLAC-SP-017 Collaboration], Phys. Rev. Lett. 33, 1406 (1974).

4. N. Brambilla, S. Eidelman, B. K. Heltsley, R. Vogt, G. T. Bodwin, E. Eichten, A. D. Frawley and A. B. Meyer et al., Eur. Phys. J. C 71, 1534 (2011).

5. W. E. Caswell and G. P. Lepage, Phys. Lett. B 167, 437 (1986).

6. G. T. Bodwin, E. Braaten and G. P. Lepage, Phys. Rev. D 51, 1125 (1995) [Erratumibid. D 55, 5853 (1997)].

7. P. Artoisenet, J. P. Lansberg and F. Maltoni, Phys. Lett. B 653, 60 (2007).

8. J. M. Campbell, F. Maltoni and F. Tramontano, Phys. Rev. Lett. 98, 252002 (2007).

9. P. Artoisenet, J. M. Campbell, J. P. Lansberg, F. Maltoni and F. Tramontano, Phys. Rev. Lett. 101, 152001 (2008).

10. G. C. Nayak, J.-W. Qiu, G. F. Sterman, Phys. Lett. B613, 45-51 (2005); Phys. Rev. D72, 114012 (2005); Phys. Rev. D 74, 074007 (2006).

11. Z.-B. Kang, Y. -Q. Ma, J.-W. Qiu, G. Sterman, in preparation.

12. S. J. Brodsky, G. P. Lepage, Adv. Ser. Direct. High Energy Phys. 5, 93 (1989).

13. B. Gong, J.-X. Wang, Phys. Rev. Lett. 100, 232001 (2008).

14. J. P. Lansberg, Eur. Phys. J. C61, 693-703 (2009).

15. M. Butenschoen and B. A. Kniehl, Phys. Rev. Lett. 106, 022003 (2011).

16. Y. Q. Ma, K. Wang and K. T. Chao, Phys. Rev. Lett. 106, 042002 (2011). 